\title{
Interrupción, intermitencia y recorte de la asistencia en la escuela secundaria
}

\author{
Mercedes Saccone, Ph. D. ${ }^{\text {a }}$ \\ Unidad Ejecutora en Ciencias Sociales Regionales y Humanidades, \\ CONICET/Universidad Nacional de Jujuy, Argentina
}

sacconemercedes@gmail.com

\section{Resumen (analítico)}

El propósito de este artículo es describir los procesos discontinuos que atraviesan las experiencias escolares de los/as jóvenes en relación con la asistencia escolar en una escuela secundaria técnica de la ciudad de Rosario (Argentina). Problemática que ha cobrado un renovado interés público y académico a partir de la sanción de la obligatoriedad de la educación secundaria. El estudio fue realizado desde un enfoque socioantropológico que recupera la tradición etnográfica de la antropología desde una perspectiva crítica. Se advierte que los procesos discontinuos identificados pueden favorecer la interrupción de la escolarización, es decir, el alejamiento de la escuela durante un período más o menos prolongado de tiempo. Pero, aunque no es total, esa lógica de discontinuidad también atraviesa las experiencias de quienes continúan cursando.

\section{Palabras clave}

Escuela secundaria, joven, asistencia escolar, interrupción/intermitencia, Argentina.

\section{Thesauro}

Tesauro de Ciencias Sociales de la Unesco.

\section{Para citar este artículo}

Saccone, M. (2022). Interrupción, intermitencia y recorte de la asistencia en la escuela secundaria. Revista Latinoamericana de Ciencias Sociales, Niñez y Juventud, 20(1), 1-29. https://dx.doi.org/10.11600/rlcsnj.20.1.4603

\section{Historial}

Recibido: 24.08.2020

Aceptado: 25.01.2021

Publicado: 10.11.2021

\section{Información artículo}

Artículo derivado de la tesis doctoral «Experiencias escolares de jóvenes en contextos de pobreza: un estudio socioantropológico en una escuela secundaria de la ciudad de Rosario (Santa Fe, Argentina)» bajo la dirección de Dra. Elena Achilli y co-dirección de Dra. Mariana Nemcovsky, Facultad de Filosofía y Letras, Universidad de Buenos Aires. Financiada a través de una Beca Interna Doctoral (01/04/2015 al 31/05/2020) del Consejo Nacional de Investigaciones Científicas y Técnicas de Argentina. Área: ciencias sociales. Subárea: antropología y educación. 


\section{Interruption, intermittence and reduction of attendance of classes in secondary school}

\section{Abstract (analytical)}

The purpose of this article is to describe the discontinuous processes that affect the school experiences of young people in relation to their attendance at a technical secondary school in the city of Rosario (Argentina). This problem has gained renewed public and academic interest due to the obligation of compulsory secondary education. The study was carried out using a socio-anthropological approach, drawing on the ethnographic tradition of Anthropology based on a critical perspective. The discontinuous educational processes that were identified can contribute to the interruption of schooling, specifically when students do not attend school for a prolonged period of time. This logic of discontinuity also crosses the experiences of those who continue with their studies.

Keywords

Secondary school, youth, educational attendance, interruption/intermittence, Argentina.

\section{Interrupção, intermitente e corte de frequência às aulas no ensino médio}

\section{Resumo (analítico)}

O objetivo deste artigo é descrever os processos descontínuos que atravessam as experiências escolares dos jovens em relação à frequência escolar em uma escola técnica secundária na cidade de Rosario (Argentina). Problema que assume um renovado interesse público e académico com a sanção da obrigatoriedade do ensino secundário. O estudo foi realizado a partir de uma abordagem socioantropológica que recupera a tradição etnográfica da antropologia de uma perspectiva crítica. Nota-se que os processos descontínuos identificados podem acabar favorecendo a interrupção da escolaridade, ou seja, a retirada da escola por um período mais ou menos prolongado. Mas, embora não seja total, notamos que essa lógica da des-continuidade também atravessa as experiências daqueles que continuam estudando.

\section{Palavras-chave}

Escola secundária, joven, frequência escolar, interrupção, intermitente, Argentina.

\section{Información autora}

[a] Licenciada en Antropología. Magíster en Ciencias en la Especialidad en Investigaciones Educativas, Instituto Politécnico Nacional (México). Doctora, Facultad de Filosofía y Letras de la Universidad de Bueno Aires, Área Antropología (Argentina). Becaria Posdoctoral Temas Estratégicos del Consejo Nacional de Investigaciones Científicas y Técnicas de Argentina, Universidad Nacional de Jujuy, Argentina. (iD 0000-0003-4705-5490. H5: 0. Correo electrónico: sacconemercedes@gmail.com 


\section{Introducción}

Ta problemática de la asistencia escolar en la educación secundaria ha cobrado Ley de Educación Nacional 26206 de 2006 (Presidencia de la Nación, 2006) y de las políticas que se han implementado con la intención de apuntalarla, sobre todo en aquellos sectores sociales donde la continuidad y finalización del nivel no se han visto garantizados.

En tal sentido, la asistencia a clases ${ }^{1}$ emerge como una dimensión central para analizar las experiencias escolares en la educación secundaria de jóvenes que habitan en espacios sociourbanos en condiciones de pobreza, objetivo del proceso de investigación más amplio en el cual se inscribe este trabajo. ${ }^{2}$ En dicha investigación, desarrollada entre los años 2014 y 2019 en la zona oeste de la localidad de Rosario (Santa Fe, Argentina), me preguntaba ¿cómo viven cotidianamente estos y estas jóvenes su paso por la escuela secundaria? ¿Qué prácticas, sentidos, relaciones y procesos configuran y permean sus experiencias escolares en estos contextos? Contextos atravesados por procesos históricos de desigualdad, ${ }^{3}$ profundizados a causa de las medidas de corte neoliberal tomadas por el

\footnotetext{
${ }^{1}$ En tanto la asistencia escolar suele referir al hecho de entrar y permanecer en la escuela, en este escrito utilizo la expresión «asistencia a clases» para remarcar que las inasistencias no siempre implican la ausencia de los y las estudiantes a la jornada escolar completa.

${ }^{2}$ Tesis doctoral en Antropología (Facultad de Filosofía y Letras de la Universidad de Buenos Aires) denominada Experiencias escolares de jóvenes en contexto de pobreza: un estudio socioantropológico en una escuela secundaria en la ciudad de Rosario (Santa Fe, Argentina).

${ }^{3}$ Las investigaciones dan cuenta de cierta continuidad histórica de un proceso de concentración espacial de las familias que viven en condiciones de pobreza en esta zona de Rosario, a partir de la década de 1980, favorecido desde las políticas sociales y de planeamiento urbano municipales y provinciales (Bernardi \& Sánchez, 2006). Proceso de «concentración geográfica de la pobreza» (Auyero, 2001, p. 22), concomitante con la creciente fragmentación y segregación que, como resultado de la agravación de las desigualdades sociales, caracterizan las grandes ciudades latinoamericanas (Prévôt-Schapira, 2001).
} 
gobierno nacional (de la alianza Cambiemos, 2015-2019), ${ }^{4}$ que afectaron tanto las condiciones de vida de gran parte de la población del pais ${ }^{5}$ como de la educación pública a la que suelen acceder los sectores sociales que viven en condiciones de pobreza. ${ }^{6}$

Con la intención de analizar las experiencias escolares de estos y estas jóvenes, a escala de sus configuraciones cotidianas, desarrollé la investigación desde un enfoque socioantropológico relacional (Achilli, 2005), inscrita en la tradición de estudios etnográficos sobre problemáticas educativas latinoamericanas. Desde este enfoque, se señala la relevancia de incorporar el nivel analítico de lo cotidiano para documentar el movimiento heterogéneo, conflictivo y contradictorio de la trama de procesos, relaciones, prácticas y sentidos que ponen en juego los sujetos, permeados y conectados por procesos más generales (Achilli, 2005; Rockwell, 1995, 2001; Rockwell \& Ezpeleta, 1985).

A partir del análisis realizado, describo ciertos procesos discontinuos que atraviesan las experiencias escolares de los y las jóvenes, específicamente en relación con la asistencia a clases; procesos que identifico como de interrupción, intermitencia y recorte de la asistencia a clases.

Sostengo que los procesos discontinuos que atraviesan la configuración cotidiana de las experiencias escolares juveniles pueden llegar a favorecer la interrupción de la escolarización, es decir, el alejamiento de la escuela durante un período más o menos prolongado de tiempo. Sin embargo, la lógica de discontinuidad también aparece en las experiencias cotidianas de las y los estudiantes que continúan cursando. Tales procesos discontinuos suponen un conjunto de prácticas, sentidos y relaciones que describo en el cruce

\footnotetext{
${ }^{4}$ Desde el inicio de su mandato (diciembre de 2015), las medidas adoptadas bajo la presidencia de Mauricio Macri han conducido a un nuevo ciclo de endeudamiento externo del sector público y una fuerte devaluación de la moneda (peso argentino); acompañados por una creciente inflación, recorte del gasto público, ajuste regresivo (deterioro del salario real, despidos y suspensiones tanto en el sector público como el privado), recesión y transferencia de ingresos hacia «distintas fracciones del poder económico, como los grandes exportadores, los principales formadores de precios, el sector financiero, las prestatarias de servicios públicos y el oligopolio petrolero» (Cantamutto \& Schorr, 2016, p. 16).
}

${ }^{5}$ De acuerdo con los datos de la Encuesta Permanente de Hogares, proporcionados por el Instituto Nacional de Estadística y Censos ([Indec], 2020), el porcentaje de hogares bajo la línea de pobreza en Argentina pasó del 17.9 $\%$ en el segundo semestre de 2017 (lo que comprende el $25.7 \%$ de las personas) al $25.9 \%$ de los hogares en el segundo semestre de 2019 (porcentaje que incluye el 35.5\% de las personas) (Indec, 2020, p. 3).

6 Ya durante el «año 2016, como producto de los ajustes y subejecuciones producidos por el nuevo gobierno, la inversión en educación se redujo al 5.8\% del PBI (...). Por otro lado, también se observa que mientras retrocede la participación global del rubro de Educación y Cultura en el PBI, crece la proporción destinada al pago de los intereses de la deuda externa» (Feldfeber et al., 2018, p. 28). 
con procesos que remiten a las condiciones de vida en contextos de pobreza urbana, las condiciones que se van imponiendo a nivel institucional y, a partir de las políticas educativas, «procesos sociales con contenidos concretos, no otorgables apriorísticamente» (Achilli, 2005, p. 17).

\section{Antecedentes de investigación y perspectiva teórica adoptada}

Los estudios sobre trayectorias escolares o educativas de los y las jóvenes de sectores populares, en tanto recorridos por el nivel medio de escolarización, refieren a que estas suelen estar marcadas por múltiples interrupciones (Binstock \& Cerrutti, 2005; Blanco et al., 2014; Bracchi \& Gabbai, 2013; Estrada, 2018; Kantor, 2000; Kessler, 2004; Montes, 2011; Montesinos \& Schoo, 2015; Terigi, 2011; Vanella \& Maldonado, 2013; Weiss, 2014). De allí que se las hayan caracterizado en términos de discontinuas, interrumpidas, inconclusas o fragmentadas. Con estos términos se suele referir a las interrupciones ${ }^{7}$ o lo que algunos denominan «abandono escolar». Se analizan la multiplicidad de factores imbricados que ejercen influencia en estos recorridos (económicos, sociales, personales, institucionales), generalmente desde lógicas extensivas o perspectivas biográficas y longitudinales (Blanco et al., 2014; Guerra, 2008; Muñoz-Izquierdo et al., 1979; Saccone, 202ob; Secretaría de Educación Pública \& Consejo para la Evaluación de la Educación del Tipo Medio Superior, 2012; Solís et al., 2014; Weiss, 2014). ${ }^{8}$ De este modo, algunos señalan la «necesidad de incorporar marcos interpretativos que asuman el carácter interseccional que define e interviene en la construcción del fracaso y del abandono escolar» (Vázquez-Recio \& LópezGil, 2018, p. 5).

Además, los especialistas remarcan la importancia de revisar los eventos previos en las trayectorias escolares (inasistencias reiteradas, bajas calificaciones, entre otros) que pueden estar indicando un «proceso de progresiva desafiliación escolar» (Blanco et al., 2014, p. 47), desapego o desenganche (Fernández-Enguita, 2011; González, 2017). Estos procesos no suelen indagarse a nivel de las experiencias escolares cotidianas de los y las jóvenes (Saccone, 2019) y, con frecuencia, son analizados en función de explicar los procesos de

\footnotetext{
${ }^{7}$ «Interrupción escolar» refiere, en términos amplios, a los eventos que implican un corte en la continuidad de la trayectoria escolar, que puede o no tornarse definitiva (Blanco et al., 2014).

${ }^{8}$ Estos aspectos son también señalados en estudios que analizan la temática de la permanencia/deserción o abandono en otros niveles y modalidades educativas (en la educación inicial, Salinas-Quiroz et al., 2017; en la universidad, Valdés-Henao, 2018; en escuelas de segunda oportunidad, Espinosa et al., 2019; Salva-Mut et al., 2016).
} 
abandono o fracaso escolar.' En otras palabras, las investigaciones mencionadas suelen dejar de lado el análisis de «las prácticas de los sujetos que protagonizan los recorridos formativos» (Santillán, 2007, p. 13) y los contenidos concretos que adquieren tales experiencias escolares, más allá de que deriven o no en interrupciones de la escolarización.

En este trabajo se entiende por experiencias escolares el conjunto de interacciones y de prácticas que desenvuelven los sujetos en los distintos ámbitos cotidianos por los que transitan -en este caso el escolar-; procesos de co-construcción que se desarrollan bajo «determinadas condiciones contextuales e históricas» (Villarreal et al., 2018, p. 3). Como señala Rockwell (1995), el hecho de asistir y estar en la escuela varias horas al día, durante años, «necesariamente deja huellas en la vida», pero el «contenido» de esa experiencia no resulta homogéneo, sino que varía de acuerdo al momento histórico, incluso «de escuela a escuela» (Rockwell, 1995, p. 13).

\section{Método}

Parto de recuperar una tradición de estudios etnográficos sobre problemáticas educativas latinoamericanas que fuera impulsada y difundida desde la década los ochenta, fundamentalmente a partir de los trabajos de Elsie Rockwell en el Departamento de Investigaciones Educativas (Cinvestav, IPN) en México, las investigaciones llevadas a cabo por Graciela Batallán y María Rosa Neufeld en la Universidad de Buenos Aires y de Elena Achilli en la Universidad Nacional de Rosario, en Argentina.

En línea con esta tradición, desarrollé la investigación, de la que es producto este escrito, desde un enfoque socioantropológico relacional (Achilli, 2005). Un abordaje que tiene en cuenta los diferentes niveles en juego para «analizar y reconstruir la propia lógica del proceso» bajo estudio (Rockwell, 2009, p. 21) y conocer la cotidianidad cuyo contenido no es evidente (Achilli, 2005).

La construcción de la información empírica se realizó a partir de un trabajo de campo intensivo y de larga duración, desarrollado entre los años 2014 y 2018 en una escuela

\footnotetext{
${ }^{9}$ Se enfatiza en el análisis del «proceso paulatino de desenganche de los aprendizajes y la vida escolar en el que entran en juego factores y situaciones múltiples y de distinta naturaleza. Un proceso que conduce a que el estudiante se aleje progresivamente de la experiencia educativa que le ofrece el centro escolar y abandone antes de finalizar su educación secundaria» (González, 2017, p. 19).
} 
técnica de nivel secundario ${ }^{10}$ en la ciudad de Rosario (Santa Fe, Argentina). A esta escuela asisten jóvenes que viven en barrios y villas cercanas. Si bien conté con el consentimiento correspondiente de los actores escolares para llevar adelante la investigación, por motivos éticos, el nombre de la escuela fue omitido y los nombres de los sujetos involucrados se modificaron para mantener el anonimato. La elección de la escuela estuvo dada por una relación laboral previa.

Para la construcción de la información recurrí a diferentes estrategias: observaciones (de clases, recreos, jornadas y actividades diversas), talleres, conversaciones informales y entrevistas (grupales e individuales) con los y las jóvenes (en promedio $45 \%$ varones y $55 \%$ mujeres $)^{11}$ de entre 13 y 26 años de edad, que asistían de primero a sexto año del turno noche. La mayoría de estos jóvenes trabaja o trabajó en algún momento, sobre todo los jóvenes varones, generalmente en condiciones precarias e informales, o tienen responsabilidades domésticas o de cuidados, generalmente de niños y niñas, tarea que suele recaer más frecuentemente en las mujeres. A su vez, con la intención de profundizar la indagación de los procesos que se desenvuelven en la cotidianidad del aula, durante un año lectivo (marzo a diciembre de 2017) hice el seguimiento a un grupo escolar (tercer año del turno noche). Por tal motivo, asistí a las clases durante una jornada completa, un día a la semana (variando de día), a lo largo de todo el año. ${ }^{12}$ A comienzos de 2017 la matrícula de este curso estaba compuesta por ocho mujeres (de 15 a 19 años; dos eran madres de niños y niñas menores de tres años y una se encontraba embarazada, siete trabajaban o

${ }^{10}$ La Ley de Educación Nacional 26206 (2006) plantea que la «Educación Secundaria es obligatoria destinada a quienes hayan cumplido con el nivel de Educación Primaria. Se divide en dos (2) ciclos: un (1) Ciclo Básico, de carácter común a todas las orientaciones y un (1) Ciclo Orientado, de carácter diversificado según distintas áreas del conocimiento, del mundo social y del trabajo» (Art. 17). La educación primaria y secundaria tienen una duración total de doce años (seis de primaria y seis de secundaria o siete y cinco respectivamente), dependiendo la jurisdicción. La secundaria de modalidad técnico-profesional posee un año más de duración, con una mayor carga horaria, dado que los títulos emitidos son habilitantes para el desempeño profesional. En la provincia de Santa Fe la estructura adoptada es de siete años de educación primaria y cinco de secundaria, con una duración de seis años para la secundaria técnica (Ministerio de Educación de la Provincia de Santa Fe, 2007).

${ }^{11}$ Esta proporción varía de acuerdo con el turno (en el turno noche la proporción de varones es mayor) y de acuerdo con el año en que realicé el trabajo de campo.

${ }_{12}$ Debido a lo intensivo de este tipo de estrategias, seleccioné uno de los cursos. Elegí trabajar en el tercer año porque se encuentra a la mitad del plan de estudios (seis años de duración), bajo el supuesto de que los primeros y últimos años estarían más atravesados por la cercanía con la escolarización primaria y la finalización del nivel secundario respectivamente. Esta estrategia tiene como limitación el que, al centrarse en un solo año escolar, se pierde la posibilidad de profundizar en las particularidades que puedan darse en otros cursos. 
habían trabajado alguna vez) y nueve varones (de 16 a 22 años; uno era padre y otro se enteró que iba a serlo durante ese año; todos se encontraban trabajando).

Aunque no con la misma profundidad, también se realizaron observaciones, talleres, conversaciones informales y entrevistas (grupales e individuales) con estudiantes que asistían a los otros turnos escolares (mañana y tarde). Considerando que las experiencias se construyen en interacción con otros, el trabajo de campo incluyó a otros sujetos (directivos, personal docente y no docente de la institución, así como funcionarios de distintos niveles del estado). Complementariamente, revisé fuentes documentales (leyes, decretos, resoluciones, documentación oficial que llega a la escuela, entre otras).

El trabajo de campo y las reflexiones que presento fueron potenciadas por mi participación en dos proyectos de investigación colectiva radiados en el Centro de Estudios Antropológicos en Contextos Urbanos, Facultad de Humanidades y Artes, Universidad Nacional de Rosario: 1) Proyecto Jóvenes y experiencias socioeducativas: un estudio antropológico de los sentidos sobre la escolarización en contextos de pobreza urbana, PID1HUM 520 (2016-2019), Secretaría de Ciencia y Tecnología de la Universidad Nacional de Rosario; 2) Proyecto Jóvenes y educación técnica: un estudio antropológico de los sentidos acerca del trabajo en la escuela técnica, Fonietp 2017 NRU 212 (2017-2018), Instituto Nacional de Educación Tecnológica, Ministerio de Educación y Deportes de la Nación. En el marco de estos proyectos se desarrolló parte del trabajo de campo en la escuela, el cual incluyó: un cuestionario con dos preguntas abiertas (¿Cómo llegaste a esta escuela? ¿ Tu familia tuvo algo que ver con esa decisión?) realizado a estudiantes de primero a sexto año del turno noche (22/06/2017, 09/o8/2017 y 15/o8/2017); los talleres mencionados (con los y las estudiantes de primero a sexto de los turnos mañana y noche en 2016 y 2017); tres entrevistas grupales (una con los y las estudiantes de tercer año, otra en cuarto y una con los y las jóvenes que asistían a quinto y sexto año del turno noche), esto en 2017; y dos entrevistas grupales con jóvenes cuya asistencia a la escuela se encontraba interrumpida, en 2018.

Desde el enfoque socioantropológico el análisis no es concebido como un momento posterior al de la construcción de la información empírica, sino que va produciéndose en simultáneo y dando lugar a la elaboración de escritos sucesivos. Un proceso recursivo en el cual «la información [empírica] es sometida — desde determinada conceptualización- a análisis crítico, a contrastaciones, a triangulaciones», a un trabajo continuo de «problematización sobre el material» (Achilli, 2005, p. 80). Todo ello fue abonando la construcción del objeto de estudio, el cual, nos dice Rockwell (2009), «toma finalmente 
la forma de un texto, ${ }^{13}$ una serie de narraciones y descripciones organizadas de tal manera que muestren ciertas relaciones de un entramado real que siempre será más complejo» (p. 75).14 Narraciones y descripciones que me permiten dar cuenta de los procesos identificados, «no a modo de formulaciones vacías que silencian a los propios protagonistas, sino reconociendo el conjunto de representaciones, significaciones y sentidos que generan los sujetos como parte de un conjunto social» (Achilli, 2005, p. 25).

\section{Resultados}

En este apartado describo los procesos discontinuos relativos a la asistencia a clases que atraviesan las experiencias escolares de los y las jóvenes, identificados a partir del análisis realizado: a) interrupciones que pueden suponer el alejamiento de los/as jóvenes de la escuela durante un período considerable de tiempo, incluso años; b) inasistencias reiteradas durante el ciclo lectivo (asistencia intermitente); y c) inasistencias a determinadas clases que no necesariamente implican ausentarse a la jornada escolar completa (procesos que recortan la asistencia a clases). Si bien se encuentran estrechamente vinculados, los separé para el análisis en tanto me permiten describir distintos aspectos que se ponen en juego a nivel de la cotidianeidad escolar.

\section{Procesos de interrupción de la escolaridad}

En principio vale mencionar que la interrupción influye en la disminución de la matrícula que posee la escuela en dos sentidos: primero, en el transcurso de los años de la

13 «La construcción del texto etnográfico se realiza mediante una serie de descripciones sucesivas» (Rockwell, 2009, p. 85). «En el trabajo de ir construyendo una descripción de cierta parte [nunca del todo] del proceso estudiado, es posible distinguir algunos procedimientos analíticos, es decir, lo que se hace con el material para transformarlo» (Rockwell, 2009, p. 85). En esta investigación, retomo: la interpretación, reconstrucción, contrastación y explicitación, propuestos por Elsie Rockwell (Rockwell, 20og). Por razones de espacio, no profundizaré su caracterización; no obstante, me interesa destacar que, desde esta perspectiva, «construir conocimiento significa dar contenido concreto a los conceptos que se elaboran; significa, además, establecer las relaciones no solo entre conceptos en abstracto, sino entre los conceptos y los contenidos empíricos provenientes de un contexto histórico en la localidad del estudio» (Rockwell, 2009, p. 92).

${ }_{14}$ Como advierte Rockwell (2009), «los conceptos designan relaciones, no denominan cosas. Las relaciones son lo que no es observable en la realidad estudiada; son, justamente, lo que se tiene que construir, lo que permite articular de manera inteligible los elementos observables (...); en el proceso analítico, se proponen relaciones que permiten integrar y hacer inteligibles algunos elementos observados» (p. 72). 
cursada, es decir, de primero a sexto año y, segundo, al interior del mismo curso, de inicio a fin del año lectivo.

Vicedirectora: Muchos empiezan. ¿Cuántos son en primer año?

Docente: 42

Vicedirectora: 42. ¿Y en sexto, Eduardo? [Estudiante de sexto año]

Eduardo: Seis.

Vicedirectora: (...) lo mismo pasa a nivel provincial. No es nuestra escuela; no es «nos pasa a nosotros». Es el $50 \%$ de las escuelas.

Docente: Los primeros años son muy numerosos y después hay un desgranamiento. (Registro de observación de jornada autoevaluación institucional, 27/10/16)

Se puede observar un contraste entre el ingreso y el egreso, ya que la escuela cuenta con cuatro divisiones de primer año, con una inscripción que suele superar 4o estudiantes en cada una de ellas, y posee solo dos divisiones de sexto año, que no llegan a contar con diez estudiantes cada una. ${ }^{15}$

Como lo han señalado investigaciones precedentes (véase Antecedentes), las condiciones de vida de los y las jóvenes y sus familias pueden favorecer la interrupción de la escolaridad. La necesidad de trabajar es uno de los aspectos señalados por los jóvenes varones (y, con menor frecuencia, por las mujeres), sobre todo por el cansancio y desgaste físico que puede producir estudiar y trabajar en condiciones precarias y jornadas extensas: «Hacía carga y descarga [en el Mercado] y terminaba muy muerto; entonces decidí abandonar» (entrevista a Alan, sexto año, o7/12/16). Aunque puede que algunos terminen interrumpiendo la asistencia a la escuela por este motivo, otros despliegan toda una serie de estrategias para poder continuar estudiando, como el cambio de turno escolar, incluso en reiteradas oportunidades: «En primer año, bueno, yo trabajaba como ayudante de albañil; entonces vine a la noche (...); después me volví a la mañana, me quedé sin trabajo, me volví a la mañana» (entrevista a Daniel tercer año, 24/o8/17). La posibilidad del cambio de turno es facilitada por las autoridades escolares, sobre todo para quienes trabajan, favoreciendo la continuidad de la escolarización.

${ }^{15}$ Los datos disponibles para el departamento Rosario «dan cuenta de 26 mil jóvenes de entre 15 y 19 años (26.7\% de esa población) que no asisten al sistema educativo y que no han logrado terminar la escuela secundaria. Poco más de la mitad de este grupo (1360o jóvenes) alcanzó a iniciarla y abandonó en algún momento del tránsito por el nivel, mientras que 7300 finalizaron la primaria, pero no iniciaron la secundaria. Y poco menos de 5000 permanece fuera de la escuela sin haber terminado el nivel primario» (Fattore \& Bernardi, 2014, p. 228). 
Las condiciones en que se viven las maternidades/paternidades en estos contextos también pueden influir en la interrupción de la asistencia a la escuela. Se suele señalar que los varones no interrumpen los estudios cuando son padres, sino que entre ellos la principal causa sería la necesidad de trabajar (Serovich, 2018). Si bien, como lo documenté, el trabajo suele ser mencionado por los jóvenes varones como uno de los motivos que más influye en la interrupción escolar, resulta importante destacar la relación que existe entre la necesidad de trabajar y la paternidad en estos contextos, en tanto tener un/a hijo/a implica muchas veces tener que ampliar la jornada laboral o buscar un trabajo para conseguir mayores recursos, restando tiempo disponible para otras actividades como la escolarización. A su vez, las condiciones en que se viven las maternidades en contextos de pobreza (la gran responsabilidad que supone el cuidado de los hijos y las hijas, el cansancio que genera, las dificultades que se pueden presentar incluso durante el embarazo) pueden favorecer la interrupción, más aún cuando, además, deben trabajar fuera del hogar: «Brisa [dejó de asistir] porque tenía un nene y era sola ella y tenía que trabajar para mantenerlo y no podía venir a la escuela y cuidarlo» (entrevista a estudiantes quinto año, 24/o8/17). Dificultades que, en caso de continuar cursando, requieren de un gran esfuerzo por parte de las jóvenes madres para sostener la asistencia a la escuela, aun con intermitencias: «Me quedé un año cuando estaba embarazada en tercero (...) y recién volví cuando él [hijo] tenía dos meses...; volví a empezar» (entrevista a Ana, cuarto año, 29/11/16). Las jóvenes diferencian entre escuelas donde «no entienden» y aquellas donde son más flexibles con el registro de las faltas y los profesores «te ayudan» (entrevista a Ana, cuarto año, 29/11/16), como la escuela en la cual realicé el trabajo de campo.

El modo en que se construyen las relaciones de pareja también puede influir en la interrupción de la asistencia a la escuela:

Ana: El año pasado, una chica pasó a quinto y dejó (...) porque se casó Entrevistadora: Pero ¿por qué dejó la escuela, porque se iba a casar?

Ana: Porque el marido no la deja venir.

Ismael: Era muy celoso. (Entrevista a estudiantes quinto año, 24/o8/17)

Si bien el análisis de tal problemática ameritaría un estudio más profundo, cabe mencionar que, al indagar sobre el tema, emergen otros procesos que también podrían estar interviniendo. Por ejemplo, algunos y algunas docentes aluden a la influencia de ciertos credos y prácticas religiosas: «Lo que me comentaron es que se casó y el chico con el que se casó es evangelista y lo que hizo fue sacarla de todos los ámbitos en que se movía 
porque consideraba que es un ámbito que es perjudicial para ella. Entonces no va más ni a la escuela ni nada» (entrevista a profesor área contable, o2/o8/17).

Las distintas violencias que atraviesan la cotidianeidad de los espacios sociourbanos donde habitan también se entrecruzan con los procesos de escolarización (Nemcovsky et al., 2020; Saccone, 2020a). Así, los jóvenes señalan que en ocasiones interrumpieron la asistencia a la escuela «por la droga..., por vaguear, por hacer cagadas ${ }^{16}[$ sic $] »$ (entrevista a Leandro, cuarto año, 10/o7/14), aspectos que vinculan con la junta ${ }^{17}$ en el barrio y en la calle.

Las interrelaciones entre pares que se construyen al interior de la escuela también son mencionadas por los y las jóvenes como motivos por los que dejaron de asistir en algún momento; en particular cuando estas se ven atravesadas por algún conflicto interpersonal: «Vanina dejó porque viene Alejandro; se pelaron, dejaron de venir los dos y después empezó a venir Alejandro solo y Vanina no» (conversación informal con Miguel, tercer año, 19/10/17). No obstante, cuando las interrelaciones que construyen son de amistad o noviazgo, pueden constituirse en un incentivo para continuar.

Las interrupciones temporales de la asistencia a la escuela también aparecen vinculadas con procesos migratorios y mudanzas al interior de la ciudad; movilidades que, por lo general, se relacionan con las condiciones de trabajo de los/as jóvenes o sus familias y, a la vez, con situaciones de violencia que se pueden producir, incluso en el ámbito familiar: «Estamos preocupados porque estas chicas no están viniendo a la escuela hace una semana porque tienen problemas en la casa; porque el padre es alcohólico y les pega. Entonces se fueron a vivir con la tía a San Lorenzo y no pueden venir a la escuela» (conversación informal con vicedirectora, o9/10/17).

La interrupción de la asistencia a una escuela no siempre implica el alejamiento de la escolarización formal. Es frecuente que los y las estudiantes opten por cambiarse a otras instituciones, como la modalidad de educación de jóvenes y adultos: «Voy a ir a un EEM$\mathrm{PA}^{18}(. .$.$) ; sí, porque esta tiene sexto y no puedo. Ahí hago cuarto y quinto» (conversación$ informal con Noelia, tercer año, 05/12/17). Ciertas investigaciones señalan que el «formato institucional que adopta el currículum para la ETP [educación técnico-profesional] de la escuela secundaria, condiciona la permanencia de los alumnos en la institución» (Ma-

\footnotetext{
${ }^{16}$ La expresión «hacer cagadas» se refiere a realizar acciones consideradas incorrectas y, a veces, ilegales.

${ }^{17}$ Grupo de pares que se frecuenta.

${ }^{18}$ Escuela de Enseñanza Media para Adultos. Instituciones de la provincia de Santa Fe destinadas a personas mayores de 18 años, con una propuesta pedagógica acelerada (tres años).
} 
turo, 2018, p. 72), destacando sobre todo la gran «carga horaria que tienen y también a las exigencias mismas de la modalidad; ${ }^{19}$ ello se ve reflejado en el egreso efectivo de los alumnos» (Maturo, 2018, p. 72).

Aunque en esta investigación documento opiniones similares, sobre todo en relación con la cantidad de años que supone el cursado en esta modalidad, la interpretación debe tensionarse con los demás aspectos que vengo describiendo. Cabe mencionar que los mayores niveles de interrupción en la escuela se producen en el primer año (que corresponde, junto al segundo, a la secundaria básica; la cual es homologable a la propuesta de cualquier otra institución sin necesidad de acreditar materias extras). Incluso, aun cuando se inscriben más de 40 jóvenes en cada división de primer año, no suele asistir esa cantidad, ni siquiera a principios de año.

El enfoque socioantropológico me permitió profundizar en el análisis de procesos a los que difícilmente podríamos acceder de guiarnos únicamente a través del análisis de los números. De acuerdo con lo que he podido registrar a partir de las observaciones y entrevistas realizadas, es común que la matrícula disminuya incluso apenas iniciado el año lectivo y a medida que este avanza. Esta disminución interanual no suele ser fácil de identificar en los registros formales de asistencia que cada institución debe cargar a través de la plataforma virtual del Ministerio de Educación, en tanto existe flexibilidad en el conteo y registro de las inasistencias y no se suele dar de baja formalmente a la/el estudiante antes de que finalice el año, esperando la posibilidad de su retorno.

Un momento significado como crítico en relación con las interrupciones es la mitad del año, especialmente luego del receso de invierno: «Después de las vacaciones de invierno..., siempre la deserción es casi 50\% de tu matrícula» (entrevista a profesor área contable, 29/11/16).

Entrevistadora: Miguel, iy Yoel?

Miguel: Lo vi hace dos semanas. Pasé con la moto y le toqué bocina; le pregunté por el Face[book] por qué no viene, que no haga lo del año pasado y no contestó (...). El año pasado iba a la misma escuela [que yo], hizo lo mismo (...). Yo iba a segundo a la mañana y él tercero a la tarde y dejó a mitad de año y se quedó. (Conversación informal con estudiantes tercer año, $02 / 08 / 17$ )

19 Como señalé en páginas anteriores, a diferencia de la escuela secundaria orientada (cinco años de duración), en la provincia de Santa Fe la educación técnica-profesional posee un plan de estudios de seis años de duración y una carga horaria mayor que supone el cursado en doble turno. 
El receso de invierno implica la no asistencia a la escuela durante dos semanas del mes de julio (a veces tres por las mesas de exámenes de materias adeudadas de años anteriores con suspensión de clases) y el retorno se vuelve difícil para algunos y algunas jóvenes. Las primeras dos semanas posteriores al receso invernal del año 2017, en el curso donde estaba realizando el seguimiento (tercero del turno noche), solo asistieron cuatro jóvenes - y con intermitencias - de los once estudiantes que asistían con cierta regularidad (registro de observación, 12/06/17).

Un docente comentaba al respecto:

La semana pasada, este grupo de tercero, que encima son de los más numerosos, solamente estuvieron viniendo cuatro chicos. Toda la escuela estaba preocupada, porque los otros cursos ya están más o menos consolidados, pero este curso que venía siendo numeroso y que es el que va a alimentar después a cuarto, quinto, sexto, porque van a seguir, les preocupó mucho. Incluso el director estaba preocupado porque solamente había cuatro chicos que estaban yendo (...). Hablamos con los chicos y nos dijeron que no, que ellos sí iban a ir, que no estaban viniendo nomás, pero que sí iban a ir. (Entrevista a profesor área contable, $02 / 08 / 17$ )

Los y las jóvenes que trabajan manifiestan llegar con mucho cansancio a ese momento del año: «[Dejé] a mitad del año; no aguantaba más» (entrevista a Alan, sexto año, 07/12/16). Además, las bajas temperaturas en dicha época, sumadas a las malas condiciones edilicias de la escuela, también influyen, más aún cuando se asiste con hijos/as pequeños a clase: «Te cansás de la escuela; levantarte temprano, hace frío» (entrevista a Fernanda, tercer año, 14/o8/14). A estos motivos, se suma la «fiaca», vagancia que produce (falta de ganas, energía), luego de un tiempo de no asistir, volver a cursar:

Javier: Llega la, llega la mitad de año y chau, dejan un montón.

Eliana: Terminan dejando.

Entrevistadora: ¿Ustedes por qué creen que dejan?

Javier: Están cansados profe (...); te da fiaca, vagancia y después cuando en el futuro dicen «cómo no termine la escuela». (Entrevista a estudiantes tercer año, o4/o8/14)

Entonces, es posible afirmar que la costumbre de asistir diariamente a la escuela puede favorecer el sostenimiento de la asistencia: «No me dan ganas de venir, pero cuando ya estoy acá, me gusta» (conversación informal con Fabricio, tercer año, 18/04/17). «Yo quería aser [sic] un empa [Eempa], pero en los lugares que fui empezaban en agosto y me 
puse a pensar y dije se me va a pasar las ganas de estudiar asta [sic] que espere agosto [sic]» (cuestionario estudiante, tercer año, 09/08/17).

Esta disminución paulatina de la matrícula se expresa en la construcción de dos imágenes distintas de la escuela: a principio del ciclo lectivo y hacia el final del año. Una escuela llena: «Cuando empieza el año la escuela está llena; el patio, los pasillos, los salones» (conversación informal con profesor área contable, o9/o8/17); y otra vacía, aburrida hacia el final: «Estamos en recreo, aunque no parezca, porque no hay nadie en la escuela» (conversación informal con profesora, 13/11/17). «¡Qué aburrimiento! [risas] No viene nadie a la escuela» (conversación informal con Paula, tercer año, 13/11/17).

Si bien escapa al objetivo de este trabajo, en estas significaciones sobre la escuela (vacía, aburrida) se pueden advertir indicios de la relevancia que adquiere la socialización entre pares en la escuela secundaria; aspecto que se vincula con el significado de la escuela como espacio de vida juvenil analizado por otros especialistas (Maldonado, 200o; Weiss, 2012).

Aunque la interrupción de la escolaridad es muy frecuente, también es posible encontrar estudiantes de esta escuela que se reincorporan al cursado. Jóvenes varones y mujeres que, luego de un tiempo de no asistir, retomaron los estudios. Aquellos y aquellas estudiantes que se alejaron de la escuela durante un tiempo y luego regresaron manifiestan que lo hicieron «porque..., me hablaron todos y es verdad, no se puede conseguir trabajo si no tenés secundario» (entrevista a Ailén, quinto año, 3o/o7/14); porque consiguieron quién cuide de sus hijos e hijas mientras asisten (entrevista a Eliana, tercer año, 04/o8/14); porque «quiero estudiar algo para no...; no tener un trabajo muy pesado en el futuro» (entrevista a Javier, tercer año, o4/o8/14) o porque «mi papá me decía que estudie...; y él aparte me apoya» (entrevista a Fabricio, tercer año, 24/o8/17). Motivos de retorno en los cuales se expresa la apropiación de una valoración positiva de la escolarización y que, a veces, aparece asociada a un proceso de cambio en la forma de pensar, significado como arrepentimiento (registro de taller primer año, 12/10/16) o rescate, como lo han documentado otras investigaciones (Benassi, 2017; Guerrero, 2006; Saccone, 2020b).

Así, las experiencias escolares de estos/as jóvenes se encuentran atravesadas por interrupciones — de mayor o menor duración temporal—en la asistencia, pero también por retornos a la escuela. Procesos que, a su vez, se entraman y se relacionan con la intermitencia y el recorte en la asistencia a clases. 


\section{«Son chicos que vienen un día sí, un día no»: procesos de asistencia intermitente}

En una clase del tercer año del turno noche, hacia mediados de octubre de 2017:

Profesor de Teoría y gestión de las organizaciones: No hay clase mañana (...). Daniela se fue porque dice que no vino el amigo [se refiere a Alejandro].

Miguel: No es el novio, son amigos; se van juntos.

Entrevistadora: ¿Y Vanina? [no está presente].

Miguel: Con Vanina se peleó.

Profesor: ¿Daniel no viene más?

Miguel: Lo vi pasar recién. Le dije: «- ¿Vas a la escuela?», «-No», me dice; parece que va a trabajar [en voz baja]; tengo que venir los jueves, si no nunca tengo Matemática.

Profesor: ¡Qué lástima!

Entra el Prosecretario con el libro de temas, mira y dice: «iUh, cuánto público!» [hay cuatro estudiantes presentes]. (Registro de observación, 19/10/17)

Como se puede observar, si bien es hacia fines del año lectivo cuando se determina con mayor claridad la escasa cantidad de los jóvenes que asiste a la escuela por los procesos que vengo describiendo, la asistencia con intermitencia a clases se produce desde inicios del ciclo lectivo. De hecho, la conformación del grupo de estudiantes de un curso resulta difícil de identificar y pueden pasar varias semanas hasta que, aun cuando sean pocos/as, conozcan los nombres de sus compañeros.

Entrevistadora: Además de ustedes dos [Daniela y Noelia] y Alejandro, ¿sigue alguien más del año pasado?

Noelia: Sí, sigue Ramiro; pero quedamos seis no más de los que empezamos (...); empezó el gordito que te hablaba a vos [se refiere a Fabricio] y el otro chico [se refiere a Yoel] y la chica de [Rosario] Central [se refiere a Vanina].

Daniela: La otra chica no está viniendo, capaz que deje. (Registro de observación tercer año, $07 / 04 / 17)$

Además, la conformación de los grupos se ve afectada por los cambios que se producen en la población de estudiantes año a año, ya que algunos no continúan cursando en esta escuela y, a la vez, se incorporan nuevos y nuevas compañeras. En ese sentido, si bien es posible que se mantenga el número de estudiantes de un curso, no significa que sean 
los mismos. Influye, también, el retraso del inicio del ciclo lectivo ${ }^{20}$ y la pérdida de días de clase, a partir del incremento de las medidas de fuerza que en los últimos años viene tomando el gremio docente ante el avance de las medidas de ajuste y disminución del presupuesto destinado a la educación pública a nivel nacional. A inicios de 2017:

El Director conversa con el Secretario acerca del paro docente y el ofrecimiento del aumento del $25 \%$ por parte de la provincia. Dicen que «no es suficiente», pero que creen que habrá docentes que acepten ${ }^{21}$ porque «es difícil continuar con las medidas de fuerza como se vienen sosteniendo con paro de 48 horas por semana». (Registro de observación, 28/o3/17)

Entre algunos/as jóvenes, el hecho de asistir con intermitencias puede implicar la no asistencia durante uno o incluso varios días de la semana. Así, una pregunta que suelen hacer los/as docentes cuando toman asistencia al inicio de la clase ${ }^{22}$ es si tal o cual joven continúa asistiendo a la escuela:

Profesor de Comunicación y comportamiento organizacional: Noelia, ¿hace mucho que no viene?

Brenda: Está viniendo poco.

Fabricio: Ya no falta nada.

Profesor: Espero que no dejen; está bien que yo vengo los lunes que siempre hay algo, pero a Noelia la vi una sola vez, a Vanina una sola vez, a Alejandro también, en este tercer trimestre; es una pena. (Registro de observación tercer año, 23/10/17)

Me interesa destacar dos cuestiones: primero, que entre los y las jóvenes se considera que un estudiante está viniendo a clases, aun cuando la frecuencia en la asistencia sea escasa, lo que puede estar expresando cierta naturalización al respecto; segundo, que esta intermitencia en la asistencia no solo favorece procesos discontinuos en relación con el trabajo con los conocimientos y la construcción de interrelaciones entre pares - aspectos en los que no profundizaré por cuestiones de espacio-, sino que dificulta incluso saber cuándo un/a joven interrumpió finalmente la escolaridad. En ese caso, la interrupción se produce al ir disminuyendo gradualmente la asistencia a clases. Pero no siempre

2o Por lo general, inicia los primeros días de marzo.

${ }^{21}$ En la provincia de Santa Fe los docentes votan en sus escuelas el rechazo o aceptación de cada ofrecimiento de aumento salarial que realiza el Ministerio de Educación.

${ }^{22}$ Si bien el control de la asistencia a la jornada escolar lo lleva la preceptora, cada docente vuelve a tomar asistencia en su clase. 
sucede de esta manera; puede ocurrir que simplemente el o la joven falte con frecuencia un mismo día, pero mantenga cierta continuidad en la asistencia a la escuela el resto de la semana.

Entrevistadora: ¿Qué tienen después?

Yanina: No sé, es la primera vez que vengo un jueves.

Ana: Yo también.

Entrevistadora: ¿Por qué?

Ana: No sé ni qué materia tenemos. (Registro taller quinto año, 22/06/17)

Cabe preguntarse cuáles son los motivos por los que, según los y las jóvenes, no asisten a clases con regularidad. En esos casos intervienen, tal como he documentado, procesos vinculados con las condiciones de vida de los estudiantes, sobre todo en relación con las responsabilidades domésticas y laborales que poseen. Asimismo, el cansancio que esas responsabilidades les producen es destacado como uno de los motivos de ausencia. En este sentido, los estudiantes manifiestan que suelen faltar «por trabajo o porque simplemente me da fiaca (...); me quedo a trabajar para tener plata» (entrevista a Marcos, quinto año, 11/o8/14). «La semana pasada vine un día nada más (...) porque yo trabajo» (entrevista a Marianela, cuarto año, 11/o8/14). «Mi viejo anda enfermo; está mi hermano [en la panadería familiar], pero no es lo mismo (...). Termino tarde y me levanto tarde» (conversación informal con Miguel, tercer año, o4/o9/17). «Tenía el nene enfermo y después internado, por eso falté (...). Tengo como siete [materias] bajas [calificaciones] en la libreta» (conversación informal con Noelia, tercer año, o3/10/17).

Entre las jóvenes madres es posible identificar la ausencia ante la necesidad de cuidar de sus hijos e hijas cuando enferman:

Ana: Y acá es fácil; acá yo falté el segundo trimestre, falté casi todo por él [hijo], porque él tiene un problema en el corazón; tiene un soplo y se enferma así y se enferma mucho, hace broncoespasmo siempre y cada dos semanas se enfermaba y no podía venir yo. Falté casi todo el segundo trimestre y después ahora vine y me puse las pilas y voy a pasar sin llevarme ninguna.

Entrevistadora: ¿Y te ayudaron los profes para que te pongas al día? Ana: Sí y con las faltas. (Entrevista a Ana, cuarto año, 29/11/16) 
También puede ocurrir que se enfermen y no puedan asistir a la escuela durante varios días: «No sé las faltas que tengo, pero me parece que más de 2o. Me enfermé, por eso falté una banda» (conversación informal con Franco, segundo año, 19/og/16).

Algunos simplemente reconocen que asisten con muy poca frecuencia: «[riéndose] Nunca vengo» (registro taller tercer año, 12/10/16).

Estefanía: Yo no sé por qué no vengo.

Entrevistadora: ¿Vos no venís? [Estefanía dice que no con un movimiento de cabeza].

Emilia: Ni pisa la escuela esta chabona. (Registro taller tercer año, 12/10/16)

A su vez, la presencia de estudiantes en la escuela los días viernes suele ser muy baja. A tal punto se encuentra naturalizada esta práctica, que se planifican las actividades teniéndola en consideración: «Si llueve lo pasamos [al taller] porque no vienen»; «Mejor el jueves porque a veces los viernes no vienen» (Conversación informal con vicedirectora, 30/05/17). Los docentes intentan convencer a los jóvenes que asistan a las clases que se desarrollan dicho día.

Profesora Taller de Marketing: Gente de tercero, no vinieron el viernes. Miércoles y viernes tenemos taller.

Noelia: No sabía que había taller

Profesora Taller de Marketing: Quedan muy poquitas clases; vengan el miércoles.

Paula: El miércoles no hay clases.

Profesora Taller de Marketing: Cierto, con más razón vengan el viernes. Les voy a dar un trabajo práctico, algo sencillo; lo empezamos a hacer aquí, pero vengan porque tuvimos muy pocas clases y si no lo hacen no van a tener nota y los voy a tener que mandar a rendir a diciembre. (Registro observación tercer año, 13/11/17)

Esta naturalización de la asistencia intermitente a la escuela me lleva a indagar en el registro y control que se realiza la institución, en tanto la normativa vigente establece un mínimo de asistencia para mantener la regularidad. ¿Qué sucede con la asistencia a la escuela en tiempos de obligatoriedad de la educación secundaria?

Dado el proceso de (s)elección por el que llegan a esta escuela jóvenes cuyos recorridos escolares previos están marcados por interrupciones, intermitencias o repeticiones de algunos años de escolaridad, la escasa matrícula que la misma posee, así como a la tendencia hacia la flexibilización que se promueve desde las políticas actuales en el país (Calamari et al., 2015; Montesinos \& Schoo, 2015; Terigi, 2011), las exigencias en torno a la 
asistencia se van tornando flexibles. En palabras de una docente: «A los pocos que vienen, que hacen el esfuerzo, los tenés que ayudar; no podés exigirles mucho» (conversación informal con profesora de Lengua, 05/12/17). De hecho, los/as estudiantes plantean que en esta escuela, a diferencia de otras, las exigencias respecto a la asistencia son menores.

Enrique: Deja un tiempo y después viene.

Cristina: Pasa, pero después te arrepentís.

Entrevistadora: ¿Por qué?

Ana: Porque pasa profe; pero después te arrepentís y volvés con todas las notas bajas.

Cristina: Es verdad, mi amiga dejó en tercer año, tenía todo aprobado el primer trimestre todo y dejó. Después pasó las semanas y se arrepintió. Pero en esa escuela no te dejaban retomar.

Entrevistadora: ¿Acá sí?

Cristina: Sí, acá vos podés faltar un mes que vos venís.

Ana: [la interrumpe] Vos venís y media falta tenés.

Cristina: Es verdad. Allá en la otra escuela no; vos faltabas una semana y ya te sacaban de la lista todo. (Registro taller quinto año, 22/o6/17)

Como se puede advertir, son tan frecuentes las inasistencias por parte de los estudiantes, que muy pocos se encontrarían en condiciones formales de mantener la regularidad. Al ser tan discontinua, la asistencia se convierte en una cuestión delicada y valorada en sí misma. Así, se vuelve en «una preocupación de toda la escuela» y «ese es un tema para la escuela, esa información..., cómo el ministerio puede ver a la escuela» (conversación informal con vicedirectora, o9/o8/17). En este sentido, para el equipo directivo y docentes, la preocupación por los números se vincula con las posibilidades concretas de sostener los puestos de trabajo. Por lo tanto, poder contar en la institución con cargos docentes, de gestión y no docentes se encuentra condicionado por la cantidad de estudiantes que posee la escuela.

$\mathrm{Al}$ respecto, es válido preguntarse: ¿qué ocurre con aquellos y aquellas jóvenes que faltan durante un tiempo o asisten con intermitencia? Si bien algunos/as pueden llegar a perder la regularidad, son muy pocos los que quedan en condición de libre. Por ejemplo, «pasa que ahora los chicos se van y después vuelven y como que no se consideran las inasistencias» (Conversación informal con vicedirectora, o9/o8/17). La consignación de las inasistencias varía según el tiempo que se haya faltado a clases, como también por las interrelaciones que se construyan con los docentes, que pueden dar lugar a negociaciones para «levantar la nota [calificación]» $\mathrm{y}$ «recuperar» el tiempo que se estuvo ausente, y 
con los preceptores, que pueden «sostenerlos con la asistencia» en cuanto al registro formal de la misma.

\section{Procesos que recortan la asistencia a clases}

Los procesos que recortan la asistencia refieren, puntualmente, a las llegadas tarde y a retirarse antes de que termine la jornada, lo que no siempre implica ausentarse la jornada escolar completa, pero sí a clases de algunas materias.

Las llegadas tarde son frecuentes sobre todo entre quienes trabajan. Las clases en el turno noche inician a las 17:30 horas. Los y las estudiantes que finalizan su jornada laboral a las 16:00 o 17:00 horas se demoran en llegar, debido a las distancias que recorren y el tiempo que les demanda prepararse para asistir a la escuela. Como lo relataba una estudiante:

Trabajo y a veces llego tarde (...) de 8 a 4 de la tarde, ponele, o no me pueden ir a buscar. Yo me tengo que venir en colectivo; ese colectivo demora 45 minutos en llegar, hasta que yo me bañe, venga y todo eso. ¿Me entendés? (Entrevista a Marianela cuarto año, 11/o8/14)

Dichos jóvenes suelen faltar a la primera hora de la jornada escolar y a veces también a la segunda. Por lo tanto, dependiendo el día, pierden las clases de una o varias materias. De este modo, algunos jóvenes no asisten durante todo el año a las clases de dichas materias. De acuerdo con los estudiantes: «Los profes te comprenden porque yo trabajo y a veces llegué un poco tarde pero me entienden [sic]» (cuestionario estudiante de tercer año, o9/o8/17). «Si llegás tarde no te dicen nada en la escuela los profes» (entrevista a Marianela, cuarto año, 11/o8/14). Vale mencionar que deciden asistir de todas formas, aun sabiendo que solo tendrán clases de algunas materias y que, probablemente, deberán recuperar o rendir dichas asignaturas en las mesas de exámenes.

Otra práctica común entre los jóvenes, la cual contribuye a recortar la asistencia a clases, es retirarse antes de que finalice la jornada escolar:

Luego de unos minutos una docente desde el patio, cerca de la sala de informática, les grita a los chicos de quinto si van a entrar. Los chicos salen, se dirigen hacia la sala de informática con sus mochilas. Cristina y Ana no entran; se retiran hacia el portón y se van de la escuela. Enrique parece dudar; se acerca a la sala de informática, ve que sus compañeras no se quedan. 
Profesor: Enrique quedate [a nosotras que estamos paradas en el patio], como son mayores [de edad] se pueden ir. (Registro de observación, 15/o8/17)

En general, los estudiantes afirman que se retiran antes porque sienten cansancio y manifiestan hacerlo con frecuencia.

Alejandro: Ahora bien, me puse las pilas.

Entrevistadora: ¿Por qué?

Alejandro: Y porque no me estaba yendo bien; me iba antes, ahora ya no. Bueno, hoy sí, pero ya no me voy siempre.

Entrevistadora: ¿Y por qué te ibas?

Alejandro: Porque me cansa; me canso de la escuela. (Conversación informal con Alejandro, cuarto año, 15/o8/18)

Dado que muchos son mayores de edad, no necesitan permiso de un mayor para retirarse. A veces avisan a sus docentes que se retirarán, otras simplemente se van durante el recreo: «Me di vuelta y se fueron dos alumnos» (conversación informal con profesora Biología, 03/10/17). Cuando avisan a sus docentes, estos y estas suelen intentar persuadirlos de lo contrario: «A mí me gustaría que te quedaras un rato más, aunque sea por la buena compañía» (registro observación tercer año, profesora Taller de Marketing, o8/11/17).

En ocasiones, el recorte de la asistencia a las clases también se puede producir en toda la escuela, ante situaciones que se viven con cierta frecuencia, como los cortes de luz debidos a las malas condiciones edilicias: «Sí, estaban contestando unas preguntas y ya pensaba que a las siete les decía que se podían ir porque no se ve y no se puede hacer más nada» (conversación informal con profesor área contable, og/o8/17).

\section{Discusión}

En este trabajo describí los procesos de interrupción, intermitencia y recortes de la asistencia a clases que atraviesan la configuración de las experiencias escolares cotidianas de jóvenes que viven en condiciones de pobreza, desde un enfoque socioantropológico que considera el proceso de investigación como el esfuerzo por relacionar distintas dimensiones de una problemática, «una perspectiva que anticipa la estructuración social no abstractamente sino en sus propias contradicciones y en su necesidad» (Achilli, 2005, 
p. 17). Procesos discontinuos que atraviesan los modos en que se vive cotidianamente el tránsito por la escuela en este contexto.

Como lo han señalado distintos autores y autoras (Binstock \& Cerrutti, 2005; Blanco et al., 2014; Bracchi \& Gabbai, 2013; Estrada, 2018; Kantor, 2000; Kessler, 2004; Montes, 2011; Montesinos \& Schoo, 2015; Terigi, 2011; Vanella \& Maldonado, 2013; Vázquez-Recio \& López-Gil, 2018; Weiss, 2014), el abordaje de los fenómenos de interrupción, abandono o fracaso escolar debe considerar su carácter multifactorial y multidimensional a fin de no reducir su complejidad.

Partiendo de estas consideraciones, y desde el enfoque adoptado, mencioné algunas de las formas concretas en que las históricas relaciones de desigualdad se expresan en las condiciones de vida de los y las jóvenes protagonistas de este estudio. Destacándose, particularmente, aquellas que emergen y se vinculan con los procesos cotidianos que documenté: las formas en que se viven las maternidades/paternidades y el cuidado de niños/as, la necesidad de conseguir recursos para aportar a la economía del hogar (por lo general insertándose o ampliando la jornada laboral), las tensiones entre los tiempos escolares y laborales, las marcas del desgaste corporal que dejan las inserciones laborales -fuertemente precarizadas - a las que suelen acceder, las migraciones o mudanzas al interior de la ciudad, entre otros, son aspectos que pueden favorecer la interrupción de la asistencia a la escuela. Procesos de interrupción que, como he documentado - y lo señalan otras investigaciones- pueden suponer el alejamiento durante un período más o menos prolongado de tiempo y, en ocasiones, el eventual retorno a la escuela (Benassi, 2017; Guerrero, 2006; Saccone, 2020b).

Si bien resultan aportes relevantes, las investigaciones mencionadas suelen enfocarse en lo que identifico como interrupciones de la asistencia. Gracias a la profundidad en el estudio de los procesos cotidianos que favorece el enfoque socioantropológico, advertí que, a su vez, las condiciones mencionadas influyen en que la asistencia se torne intermitente durante el ciclo lectivo, lo que puede implicar la no asistencia a clases a uno o incluso a varios días de la semana, aspecto que difícilmente pueda identificarse solo a partir del análisis de los registros formales. De hecho, pude observar que, son tan frecuentes las inasistencias a la escuela por parte de estos estudiantes, que muy pocos se encontrarían en condiciones formales de mantener la regularidad.

Por último, identifiqué y describí los procesos que recortan la asistencia, prácticas como llegar tarde y retirarse antes que implican faltar a clases de algunas materias, generalmente aquellas que se dictan al inicio o al final de la jornada escolar. 
Estos procesos discontinuos van interrumpiendo, tornando intermitente y recortando el tiempo que los jóvenes están en la escuela, incluso de quienes continúan cursando, cuyas experiencias no siempre son incorporadas en los análisis que se focalizan en el abandono o fracaso escolar.

Tales procesos se producen, entonces, en el cruce de las condiciones de vida de estos y estas jóvenes, pero también de las condiciones que se van imponiendo a nivel institucional y de las políticas educativas. De este modo, los procesos discontinuos que describí se vinculan y se entraman con la tendencia - presente en las políticas educativas- hacia la flexibilización de aspectos como el control y registro de la asistencia a clases o de la presencialidad que, como destacan algunas investigaciones (Calamari et al., 2015; Montesinos \& Schoo, 2015; Terigi, 2011), puede favorecer la continuidad de la escolarización aun con interrupciones e intermitencias. Sin embargo, y al mismo tiempo, se corre el riesgo de reproducir desiguales formas de acceso a los conocimientos. Aspecto no abordado específicamente en este escrito, pero que alerta respecto a lo que es posible enseñar y aprender en determinadas condiciones contextuales, procesos analizados en otros países por autores como Bonal y Tarabini (2013), quienes nos recuerdan la complejidad que asumen las relaciones entre educación y pobreza, así como los peligros de caer en análisis que simplifiquen la explicación de estos fenómenos.

Los procesos discontinuos que identifiqué pueden llegar a favorecer entre algunos y algunas jóvenes la interrupción de la escolarización, es decir, el alejamiento de la escuela durante un período más o menos prolongado de tiempo (semanas, meses, incluso años), que eventualmente puede tornarse definitivo. Pero esa lógica de discontinuidad también atraviesa las experiencias de quienes continúan cursando, dando lugar a relaciones, prácticas y sentidos heterogéneos - y algunas contradictorias- que, al tiempo que van generándola, también la tensionan.

En síntesis, considero que la posibilidad de documentar en profundidad las experiencias escolares de jóvenes a nivel de los cotidianos escolares, escasamente exploradas, permite complementar y complejizar aquellos estudios de corte extensivo existentes. Estos conocimientos pueden ser retomados en la generación de propuestas y políticas orientadas a la educación secundaria obligatoria, que recuperen las expectativas, vivencias y saberes de la población estudiantil y favorezcan procesos de escolarización secundaria más equitativos, en tanto los modos de estar en la escuela que describí ponen en evidencia las grandes desigualdades que se esconden detrás de los porcentajes de acceso al nivel. Asimismo, quedan pendientes para futuros análisis los cruces y relaciones que se producen 
entre los procesos discontinuos en la asistencia a clases y el trabajo cotidiano con los conocimientos en estas condiciones.

\section{Agradecimientos}

El desarrollo de la investigación de la que deriva este trabajo contó con el apoyo financiero de una beca interna doctoral (2015-2020) del Consejo Nacional de Investigaciones Científicas y Técnicas de Argentina. Agradezco a mi directora, Dra. Elena Achilli, y codirectora, Dra. Mariana Nemcovsky, así como a las compañeras de los proyectos de investigación Jóvenes y experiencias socioeducativas: un estudio antropológico de los sentidos sobre la escolarización en contextos de pobreza urbana (2016-2019) (financiado por la Secretaría de Ciencia y Tecnología de la Universidad Nacional de Rosario) y Jóvenes y educación técnica: un estudio antropológico de los sentidos acerca del trabajo en la escuela técnica, Fonietp 2017 NRU 212 (2017-2018), financiado por el Instituto Nacional de Educación Tecnológica, Ministerio de Educación y Deportes de la Nación. Finalmente, este estudio no habría podido realizarse sin la participación y generosidad de los y las estudiantes, directivos y docentes de la escuela; a ellos y ellas todo mi agradecimiento.

\section{Referencias}

Achilli, E. (2005). Investigar en Antropología Social: Los desafíos de transmitir un oficio. Laborde Editor.

Auyero, J. (2001). Introducción: claves para pensar la marginación. En L. Wacquant (Ed.), Parias urbanos: marginalidad en la ciudad a comienzos del milenio (pp. 9-32). Manantial.

Benassi, E. (2017). «Rescatate y trabajá»: el valor-trabajo en las experiencias de vida de jóvenes de sectores populares [Ponencia]. $13^{\circ}$ Congreso Nacional de Estudios del Trabajo «El trabajo en conflicto: dinámicas y expresiones en el contexto actual»

Bernardi, G., \& Sánchez, S. (2006). Procesos de concentración espacial de la pobreza urbana: retratos de una configuración. En E. Achilli, E. Cámpora, L. Giampani, M. Nemcovsky, S. Sánchez, \& J. Shapiro (Eds.), Memorias y experiencias urbanas (pp. 21-38). UNR Editora.

Binstock, G., \& Cerrutti, M. (2005). Carreras truncadas: el abandono escolar en el nivel medio en la Argentina. Unicef. 
Blanco, E., Solís, P., \& Robles, H. (2014). Caminos desiguales: trayectorias educativas y laborales de los jóvenes en la Ciudad de México. INEE; El Colegio de México.

Bonal, X., \& Tarabini, A. (2013). De la educación a la educabilidad: una aproximación sociológica a la experiencia educativa del alumnado en situación de pobreza. Praxis Sociológica, (17), 67-88.

Bracchi, C., \& Gabbai, M. I. (2013). Subjetividades juveniles y trayectorias educativas: tensiones y desafíos para la escuela secundaria en clave de derecho. En C. Kaplan (Ed.), Culturas estudiantiles: sociología de los vínculos en la escuela (pp. 23-44). Miño y Dávila.

Calamari, M., Saccone, M., \& Santos, M. (2015). Procesos de «flexibilización» como clave para la «inclusión socioeducativa»: un análisis del Plan «Vuelvo a estudiar» en la provincia de Santa Fe. Revista de La Escuela de Antropología, (21), 47-70. https:// doi.org/10.35305/revistadeantropologia.voixxi.20

Cantamutto, F., \& Schorr, M. (2016). El gobierno de Macri: ajuste regresivo, nuevo ciclo de endeudamiento externo y cuantiosas transferencias de ingresos al poder económico. En J. Marchini, \& E. Lucita (Eds.), ¿A dónde va la economía del gobierno de Macri? (pp. 15-17). Fundación Rosa Luxemburgo.

Espinosa, O., González, L. E., Castillo, D., \& Neut, S. (2019). Condicionantes de la retención estudiantil en «escuelas de segunda oportunidad» en Chile. Revista Latinoamericana de Ciencias Sociales, Niñez y Juventud, 17(2), 1-27. https://doi.org/g476

Estrada, M. J. (2018). Abandono escolar en la educación media superior de México: políticas, actores y análisis de casos. Universidad de Guanajuato.

Fattore, N., \& Bernardi, G. (2014). Programa joven de inclusión socioeducativa. Unicef; Universidad Nacional de Rosario.

Feldfeber, M., Puiggrós, A., Robertson, S., \& Duhalde, M. (2018). La privatización educativa en Argentina. Internacional de educación; Ctera. https://bit.ly/2Yxk8SA

Fernández-Enguita, M. (2011). Del desapego al desenganche y de éste al fracaso escolar. Propuesta Educativa, (35), 85-94.

González, M. T. (2017). Desenganche y abandono escolar, y medidas de re-enganche: algunas consideraciones. Profesorado. Revista del Currículum y Formación del Profesorado, 21(4), $18-37$.

Guerra, M. I. (2008). Trayectorias escolares y laborales de jóvenes de sectores populares: un abordaje biográfico [Tesis doctoral]. Cinvestav.

Guerrero, M. E. (2006). El punto de retorno: una experiencia de bachillerato universitario. Revista Mexicana de Investigación Educativa, 11(29), 483-507. 
Instituto Nacional de Estadística y Censos. (2020). Incidencia de la pobreza y la indigencia en 31 aglomerados urbanos. https://bit.ly/3wpyj8J

Kantor, D. (2000). La escuela secundaria desde la perspectiva de los jóvenes con trayectorias escolares inconclusas [Informe final de investigación]. Secretaría de Educación; Gobierno de la Caba.

Kessler, G. (2004). Trayectorias escolares de jóvenes que cometieron delitos contra la propiedad con uso de violencia. Universidad de San Andrés. https://bit.ly/3bQrjZ5

Maldonado, M. (200o). Una escuela dentro de otra escuela: un enfoque antropológico sobre los estudiantes secundarios en una escuela pública de los noventa. Editorial Universitaria de Buenos Aires.

Maturo, Y. (2018). El derecho a la educación técnico profesional. efectos de la dinámica exclusión incluyente en una escuela técnica de Córdoba. Educación, Formación e Investigación, 4(7), 55-79.

Ministerio de Educación de la Provincia de Santa Fe. (2007). Decreto 2885. Autor.

Montes, N. (2011). Trayectorias educativas y laborales: un cruce desde la percepción de estudiantes de nivel medio. En G. Tiramonti, \& N. Montes (Eds.), La escuela media en debate: problemas actuales y perspectivas desde la investigación (pp. 113-127). Manantial.

Montesinos, M. P., \& Schoo, S. (2015). Política de revinculación y terminalidad escolar: reflexiones en torno a los abordajes contemporáneos orientados a universalizar la educación secundaria.

Muñoz-Izquierdo, C., Rodríguez, P. G., Restrepo, P., \& Borrani, C. (1979). El síndrome del atraso escolar y el abandono del sistema educativo. Revista Latinoamericana de Estudios Educativos, 35(3-4), 1-60.

Nemcovsky, M., Bernardi, G., Saccone, M., López-Fittipaldi, M., Calamari, M., Debonis, F., Dobry, M., Maiolino, E., Martínez, N., \& Santos, M. (2020). Jóvenes y escuela secundaria: un estudio antropológico en contextos de pobreza urbana y distintas violencias.

Laborde Libros.

Presidencia de la Nación [Argentina]. (2006). Ley de Educación Nacional 26 206. Autor. Prévôt-Schapira, M.-F. (2001). Fragmentación espacial y social: conceptos y realidades. Perfiles Latinoamericanos, 9(19), 33-56.

Rockwell, E. (1995). La escuela cotidiana. Fondo de Cultura Económica.

Rockwell, E. (2001). Caminos y rumbos de la investigación etnográfica en América Latina. Cuadernos de Antropología Social, (13), 53-64.

Rockwell, E. (2009). La experiencia etnográfica: historia y cultura en los procesos educativos. Paidós. 
Rockwell, E., \& Ezpeleta, J. (1985). La escuela: relato de un proceso de construcción inconcluso. En Educação na América Latina, Os modelos teóricos e realidade social (pp. 151-172). Cortez Editora.

Saccone, M. (2019). Nocturnidad en la educación secundaria técnica: análisis de una experiencia en la provincia de Santa Fe (Argentina). Revista Educación, Política y Sociedad, 4(1), 109-123.

Saccone, M. (2020a). Experiencias escolares de jóvenes en contextos de pobreza: un estudio socioantropológico en una escuela secundaria en la ciudad de Rosario (Santa Fe, Argentina). [Tesis de doctorado]. Universidad de Buenos Aires.

Saccone, M. (2020b). La asistencia a clases de los estudiantes en la educación media superior: aportes desde una investigación etnográfica en la Ciudad de México. Revista Latinoamericana de Estudios Eduativos, 5o(2), 55-88. https://doi.org/g48b

Salinas-Quiroz, F., Silva, P., Cambón, V., \& Fraga, S. (2017). Asistencia intermitente y deserción en educación inicial: testimonios de madres uruguayas. Revista Latinoamericana de Ciencias Sociales, Niñez y Juventud, 15(2), 913-925.

Salva-Mut, F., Nadal-Cavaller, J., \& Melià-Barceló, M. A. (2016). Itinerarios de éxito y rupturas en la educación de segunda oportunidad. Revista Latinoamericana de Ciencias Sociales, Niñez y Juventud, 14(2), 1405-1419.

Santillán, L. (2007). Trayectorias educativas y cotidianeidad: una etnografía del problema de la educación y la experiencia escolar en contextos de desigualdad [Tesis doctoral]. Universidad de Buenos Aires.

Secretaría de Educación Pública \& Consejo para la Evaluación de la Educación del Tipo Medio Superior. (2012). Reporte de la Encuesta Nacional de Deserción en la Educación Media Superior. https://bit.ly/3kie1JA

Serovich, L. (2018). Escuelas de educación media para ¿adultos? La juvenización de las EEMPA en la ciudad de Santa Fe. Itinerarios Educativos, 11, 167-183.

Solís, P., Leal, A., \& Brunet, N. (2014). Abandono escolar del primer semestre de la generación 2013-B del Colegio de Bachilleres. Dirección General del Colegio de Bachilleres.

Terigi, F. (2011). Sobre la universalización de la escuela secundaria: la enseñanza como problema. https://bit.ly/3mUmQL6

Valdés-Henao, C. (2018). Deserción universitaria: entre desvinculaciones institucionales y búsqueda de sentidos de vida. Revista Latinoamericana de Ciencias Sociales, Niñez y Juventud, 16(1), 331-344. https://doi.org/10.1160o/1692715x.16120 
Vanella, L., \& Maldonado, M. (2013). Programa de Inclusión y Terminalidad de la Educación Secundaria para Jóvenes de 14 a 17 años (PIT). Córdoba (Argentina). Unicef; Universidad Nacional de Córdoba.

Vázquez-Recio, R., \& López-Gil, M. (2018). Interseccionalidad, jóvenes «sin-sistema» y resistencia: una mirada diferente del fracaso/abandono escolar. Revista Brasileira de Educação, 23, 1-15. https://doi.org/10.1590/s1413-24782018230094

Villarreal, M. C., Greca, V., \& Achilli, E. (2018). Políticas públicas e interculturalidad en Argentina: un análisis de experiencias formativas Qom y Mocoví en distintos espacios de la provincia de Santa Fe. $56^{\circ}$ Congreso Internacional de Americanistas. Universidad de Salamanca, Salamanca, España.

Weiss, E. (2012). Los estudiantes como jóvenes. el proceso de subjetivación. Perfiles Educativos, 34(135), 134-148.

Weiss, E. (2014). El abandono escolar en la educación media superior. Instituto Belisario Domínguez. 